\title{
Efficacy of intravenous dexamethasone for the prevention of vomiting associated with intrathecal chemotherapy and ketamine sedation in children: a randomized, double-blinded, crossover, placebo- controlled trial
}

\author{
Chanchai Traivaree ${ }^{\mathrm{a}}$, Kitti Torcharus ${ }^{\mathrm{a}}$, Rachata Lumkul ${ }^{\mathrm{a}}$, Chulalak Komoltrib, Somrat Charuluxananan ${ }^{\mathrm{c}}$ \\ ${ }^{a}$ Division of Haematology/Oncology, Department of Pediatrics, Phramongkutklao College of Medicine, \\ Bangkok 10400; ${ }^{b}$ Division of Clinical Epidemiology, Faculty of Medicine, Mahidol University, Bangkok \\ 10700; ${ }^{\circ}$ Department of Anesthesiology, Faculty of Medicine, Chulalongkorn University, Bangkok 10330, \\ Thailand
}

\begin{abstract}
Background: Chemotherapy-induced nausea and vomiting remains a significant problem for children with leukemia. There is limited evidence to support using prophylactic antiemetic prior to the administration of intrathecal chemotherapy.

Objective: Determine whether vomiting and nausea associated with intravenous ketamine and intrathecal chemotherapy may be reduced by the addition of prophylactic dexamethasone in children.

Method: A randomized, double-blinded, crossover, placebo-controlled trial was completed in 33 children receiving intrathecal chemotherapy with methotrexate and ketamine sedation at Pharmongkutklao Hospital. Patients were randomly assigned in a double-blinded fashion to receive one of two interventions during the first period, either an infusion of normal saline or intravenous dexamethasone at $0.25 \mathrm{mg} / \mathrm{kg} / \mathrm{dose}$. Each patient acted as his or her own control, and each patient was studied at two time-points.

Results: Period effect, sequence effect, and carry over effect were not demonstrated. The absolute risk reduction of vomiting was significantly greater after infusion of dexamethasone than after placebo at $33.3 \%(p=0.02)$. The number needed to treat was three to prevent one episode of vomiting. Fifteen patients in the treatment group reported nausea versus 26 patients in the placebo group $(p=0.007)$. In the group of patients treated with dexamethasone, five required antiemetic vs. 16 of those receiving placebo $(p=0.02)$. There was no complication from dexamethasone.

Conclusion: Intravenous dexamethasone reduced vomiting associated with intrathecal chemotherapy and ketamine sedation, without significant side-effects. It may be recommended a reasonable option before intrathecal chemotherapy.
\end{abstract}

Keywords: Dexamethasone, intrathecal chemotherapy, ketamine, vomiting

Acute leukemia is the most common malignancy affecting the pediatric population. The treatment includes systemic chemotherapy and intrathecal chemotherapy, which reduces the frequency of overt relapse of acute leukemia in the central nervous

Correspondence to: Chanchai Traivaree, MD, Division of Haematology/Oncology, Department of Pediatrics, Phramongkutklao College of Medicine, Bangkok 10400, Thailand. E-mail: ctrivaree@yahoo.com system [1]. The schedule of intrathecal chemotherapy administration depends on individual protocols, varying from weekly during the induction phase to every three months during the maintenance phase [2]. Standard intrathecal chemotherapy is given in dosages according to patient age and risk for relapse. The procedure frequently results in moderate nausea and vomiting typically beginning approximately two to three hours after intrathecal chemotherapy and rarely lasting longer than 24 hours. 
Although intrathecal chemotherapy administration is a low risk procedure, it is uncomfortable, and children usually require sedation while undergoing the procedure. Sedation helps to reduce the child's movements during the procedure and decreases anxiety and pain. When intrathecal chemotherapy is combined with ketamine sedation, there is likely postprocedure nausea and vomiting within 24 hours. Ondansetron is commonly used because of its efficacy and safety for preventing nausea and vomiting.

Many publications have confirmed that ondansetron significantly reduces the incidence of nausea and vomiting either following intrathecal chemotherapy or intravenous ketamine sedation in children [3, 4]. However, the high cost of ondansetron has been a significant factor to limit its routine prophylactic use at present. Dexamethasone is another option which has been shown to be effective as a prophylactic antiemetic agent [5]. However, there is no data for using dexamethasone alone for prevention for nausea and vomiting in children receiving ketamine sedation and intrathecal chemotherapy.

In this study, we assessed the efficacy of dexamethasone for antiemetic effect in children receiving ketamine sedation for lumbar puncture with intrathecal chemotherapy administration.

\section{Materials and methods}

A convenience sample of children was enrolled in a randomized, double-blind, crossover, placebocontrolled trial conducted between March and September 2010 at Pharmongkutklao Hospital at Bangkok. Parental-informed consent was obtained before participation into the study. The study was approved by the Ethics Committee of Pharmongkutklao Hospital.

All children between 2-15 years with diagnosed acute lymphoid or myeloid leukemia who required intravenous ketamine for sedation and lumbar puncture for intrathecal chemotherapy were eligible for enrollment into the study. We only included patients during consolidation phase and maintenance phases of treatment. Patients were excluded if they received other chemotherapy within 24 hours of the treatment. We modified the treatment such that intrathecal chemotherapy which was given on separate days from other systemic chemotherapy as not to affect the primary outcome. We instructed patients to withhold oral chemotherapy the evening prior or the day of the planned procedure. In addition, efforts were made to move systemic chemotherapy to the day after the planned procedure. The exclusion criteria included patients with a known allergy to ketamine or dexamethasone, contraindications for ketamine or dexamethasone such as hypertension or hyperglycemia, concurrent nausea and vomiting, and patients who had received ondansetron or other antiemetic before the procedure.

Patients were randomly assigned to receive one of two interventions during the first period in a doubleblinded fashion. Simple randomization was performed by using a computer generated randomized type of treatment table in a downward direction. Patients received one of two options during the first period: dexamethasone $(0.25 / \mathrm{mg} / \mathrm{kg} /$ dose, maximum single dose $8 \mathrm{mg}$ ) (5 mL) (Treatment A) or normal saline solution iv (Treatment B, placebo). Both groups received the same amount of ketamine for sedation (1mg/kg iv, maximum single dose 50mg). Given the crossover design, the treatment was reversely assigned during the second period (A to B, B to A). The interval between the two treatment periods was two weeks to three months, allowing a washout period for intrathecal chemotherapy and the effect of each treatment. For allocation concealment, the type of treatment was stored in sequentially numbered, sealed, opaque envelopes. The envelopes were opened after consent was obtained. The study drug (either dexamethasone or placebo) along with ketamine were administered immediately before the induction of sedation and prior to lumbar puncture and administration of intrathecal chemotherapy.

\section{Outcome measurements}

The primary outcome was the presence (yes/no) of vomiting or retching during the 24 hours following the intrathecal chemotherapy. The secondary outcome was severity of nausea, rated as none, mild, moderate, or severe. This was evaluated at hours 3, 6, 12, and 24 following the procedure. Use of ondansetron in hospital or at home for rescue vomiting was also recorded.

Patients were observed for a minimum of two hours in the recovery room. Those who had vomiting following the procedure were given ondansetron 0.15 $\mathrm{mg} / \mathrm{kg} /$ dose (max $8 \mathrm{mg}$ ) intravenously. Children without an intravenous line were offered oral ondansetron at dose of $4 \mathrm{mg}$ (for children 2-10 years) or $8 \mathrm{mg}$ (for children $>10$ years). 
At discharge, parents were asked to observe their children for the remaining 22 hours and observe for any outcomes. Parents were given an antiemetic drug (ondansetron) to give their children in case of vomiting. Parents were asked to record all vomiting episodes, rate the severity of nausea and to indicate whether they gave ondansetron to their child at home.

Parents were telephoned at home by a research coordinator on the day of the procedure and the following day to assess outcomes.

\section{Operational definitions}

Vomiting was defined as the forceful expulsion of the contents of the stomach through the oral or nasal cavity [3]. Retching was defined as the attempt to vomit without bringing out anything up [3]. Nausea was defined as an unpleasant sensation experienced in the back of the throat and the epigastrum that may or may not culminate in vomiting [3]. The degree of nausea following the administration of intrathecal chemotherapy was scored according that developed and used previously by Holdsworth et al. [6] as follows: $0=$ none (no symptoms); $1=$ mild (can eat and drink well), $2=$ moderate (cannot eat but drink some); $3=$ severe (cannot eat and drink), respectively. In this study, the carryover effect is defined as the persistence of the effect of the first intervention extending over its period of application to influence the result of a subsequent intervention. The period effect is defined as the change of responses due to the difference between the first and second interventions. The sequence effect is defines as the difference in effect due to the order (dexamethasoneplacebo or placebo-dexamethasone) in which the intervention are given produced [7].

\section{Statistical analysis}

A power calculation was based on pilot data $(\mathrm{n}=10)$ indicating that vomiting would occur in $20 \%$ children receiving dexamethasone prophylaxis and $60 \%$ of those receiving placebo. Thus, 29 participants were needed to detect a significant difference between the two treatments with a two-sided test (type I error of $5 \%$ and $80 \%$ power). At the end of the study, we analyzed the outcomes by carry-over, period and sequence treatment effects by Fisher's exact test. We used Mcnemar's test for binary and ordinal outcomes using a significance level at $\mathrm{p}<0.05$ [8]. The statistical analysis was performed using by SPSS version15 software.

\section{Results \\ Patient characteristics}

Thirty-four children diagnosed with acute leukemia were enrolled into the study. They were randomly and equally allocated in sequence into two groups. One child was withdrawn from the study as no ketamine sedation was used during the second period. Therefore, 33 children (17 boys and 16 girls ranging from 2.2 to 14.2 years old, mean 5.8 years) completed the cross-over study. All were considered evaluable and included in the analysis. Characteristics of the enrolled patients are listed in Table $\mathbf{1}$.

The average ketamine dose used for sedation was $1.05 \mathrm{mg} / \mathrm{kg}$ (range: $0.83-1.25$ ) in placebo group and $1.07 \mathrm{mg} / \mathrm{kg}$ (range: 0.86-1.20) in the treatment group. The average duration of sedation time was 8.2 minutes (range: 6-10) in placebo group and 7.6 minutes (range: $5-9$ ) in the treatment group. No patient required more than one dose of ketamine sedation during the procedure. The median time between treatment periods for washout in the dexamethasone-placebo sequence group was three months (range: 0.5-4.5 months) and in placebo-dexamethasone sequence group was three months (range: 0.5-4 months).

Table 1. Baseline patient characteristics

\begin{tabular}{ll}
\hline Total & 33 patients \\
Gender & 17 boy, 16 girl \\
Age (year) & Average: 5.8, range: $2.2-14.2$ \\
Weight $(\mathrm{kg})$ & $20.65+8.88$ \\
Height $(\mathrm{cm})$ & $109.57+17.72$ \\
Diagnosis & 28 B cell ALL*, 4 T cell ALL, 1 AML* \\
Risk of disease & 22 standard risk, 11 high risk \\
Type of IT* chemotherapy & 32 MTX $^{*}, 1$ ARA-C* \\
Phase of Treatment & 31 Maintenance, 2 Consolidation
\end{tabular}

IT*: Intrathecal chemotherapy, ALL*: acute lymphoblastic leukemia, AML*: acute myeloid leukemia MTX*: methotrexate, ARA-C*: cytosine arabinoside 


\section{Carryover, period, and sequence effects for primary outcome}

There was no carryover effect for the primary outcome. Only one patient had vomiting in both treatment periods whereas thirteen children (39.4\%) had no vomiting in either treatment period as shown in Table 2.

The primary outcome, presence of vomiting, in either treatment period or treatment sequences was not statistically different by Fisher's exact test $(p=1.00)$. There was no period and sequence effect on the primary outcome. A comparison in both periods showed 11 patients with vomiting in the first period compared with 10 patients with vomiting in the second period. A comparison of both sequences revealed 11 patients with vomiting in the dexamethasone-placebo sequence compared with 10 patients with vomiting in the placebo-dexamethasone sequence.

\section{Primary outcome}

There were 21 episodes of vomiting from 66 treatment periods among 33 patients (31.8\%). Five episodes of vomiting occurred in the dexamethasone group (15.2\%), and 16 episodes occurred in the placebo group (48.5\%). There was a statistically significant difference between the two groups $(\mathrm{p}=0.02)$ (Table 3).

The absolute risk reduction for vomiting in the dexamethasone group was 33.3\% (95\% confidence interval (CI): $17.6 \%$ to $49 \%$ ) compared to placebo. Approximately three patients (95\% CI: 2.0 to 5.7) would need to be treated to prevent one patient from vomiting following intrathecal chemotherapy and ketamine sedation. There were significant differences among the different age groups and between genders. The vomiting group included significantly younger children than the non-vomiting group $(4.65+1.48$ years vs. $7.61+3.58$ years, $\mathrm{p}=0.002)$ and girls had a significantly lower incidence of vomiting than boys (37.5\% vs. $82.4 \%$, respectively, $\mathrm{p}=0.01$ ). However, the average age in girls was $7.12+3.4$ years and in boys was $4.59+1.57$ years $(\mathrm{p}=0.01)$. In stratified analysis among only boys and only girls, younger age continued to be associated with increased vomiting in girls, but no difference in boys. In girls, the younger age was $4.8+0.9$ years and $8.5+3.61$ years in vomiting group and non-vomiting group, respectively $(\mathrm{p}=0.01)$. In boys, the younger age was $4.6+1.7$ years and $4.6+0.97$ years in vomiting group and in non-vomiting group, respectively $(\mathrm{p}=0.95)$. The average time after procedure to vomiting in the treatment group and placebo group were 54 minutes (range: 5-110) and 57 minutes (range: 1-195), respectively.

\section{Secondary outcome}

Severity of nausea was evaluated during the first $3,6,12$, and 24 hours following the procedure according to nausea scale (0-3). Twenty-eight patients had experienced nausea at least once in 24 hours. Within three hours, nausea was significantly different between dexamethasone compared with placebo. In particular, 15 patients in the dexamethasone group had nausea versus 26 patients in the placebo group $(p=0.007)$ (Table 3$)$. The severity of nausea distributed among the 66 treatment periods (33 patients) is shown in Table 4.

Table 2. Outcome in each sequence of treatment

\begin{tabular}{lcccc}
\hline Outcome & $\begin{array}{c}\text { (Novomit, } \\
\text { Novomit) }\end{array}$ & $\begin{array}{c}\text { (Novomit, } \\
\text { Vomit) }\end{array}$ & $\begin{array}{c}\text { (Vomit, } \\
\text { No Vomit) }\end{array}$ & (Vomit, Vomit) \\
\hline Dexa-placebo sequence & 7 & 8 & 2 & 1 \\
Placebo-dexa sequence & 6 & 2 & 7 & 0 \\
\hline
\end{tabular}

Carryover effect test in dexa-placebo and placebo-dexa sequence ( $\mathrm{p}=1.00)$, Fisher's exact test.

Table 3. Effect of $0.25 \mathrm{mg} / \mathrm{kg}$ of intravenous dexamethasone before lumbar puncture in each outcome

\begin{tabular}{lccccc}
\hline & $\begin{array}{c}\text { Treatment group } \\
(\mathbf{n = 3 3 )}\end{array}$ & $\begin{array}{c}\text { Placebo group } \\
\mathbf{( n = 3 3 )}\end{array}$ & $\begin{array}{c}\text { P-value } \\
\text { (McNemar's test) }\end{array}$ & $\begin{array}{c}\text { Difference, \% } \\
\text { (95\%CI) }\end{array}$ & NNT*(95\%CI) \\
\hline Vomiting & 5 & 16 & 0.02 & $33,(18-49)$ & $3(2-5.6)$ \\
Nausea & 15 & 26 & 0.007 & $33,(15-51)$ & $3(2-6.6)$ \\
Ondansetron required & 5 & 16 & 0.02 & $33,(18-49)$ & $3(2-5.6)$ \\
\hline
\end{tabular}

$\mathrm{CI}=$ confidence interval 
Table 4. Severity of nausea within three hours in their own control

\begin{tabular}{cllccc}
\hline Severity of nausea* & \multicolumn{2}{c}{ Placebo group } & Total \\
\cline { 2 - 5 } & $\mathbf{0}$ & $\mathbf{1}$ & $\mathbf{2}$ & $\mathbf{3}$ & \\
\hline Dexamethasone group 0 & 5 & 2 & 7 & 4 & 18 \\
1 & 0 & 3 & 5 & 3 & 11 \\
2 & 2 & 1 & 0 & 0 & 3 \\
3 & 0 & 0 & 1 & 0 & 1 \\
Total & 7 & 6 & 13 & 7 & 33
\end{tabular}

Severity of nausea within three hours in the dexamethasone versus placebo group $(\mathrm{p}=0.017)$. Mcnemar's test. *Severity of nausea: $0=$ no symptoms, $1=$ can eat and drink well, $2=$ cannot eat but drink some, $3=$ cannot eat and drink

Analysis with McNemar's test showed a statistically significant difference between the results of the two groups on the nausea scale $(p=0.017)$. There were 41 episodes of nausea (mild and higher) in 66 treatments (62.1\%) among 33 patients. However, five patients experienced nausea at six hours and one at 12 hours following therapy. All of them were in the placebo arm.

Five patients (15.2\%) in the dexamethasone group and $16(48.5 \%)$ in the placebo group received ondansetron rescue after the procedure $(\mathrm{p}=0.02)$ (Table 3). Five patients who received dexamethasone required only one dose of ondansetron (100\%). Among those who received placebo, 11 required one dose (68.7\%), four patients two doses, and one patient three doses.

\section{Safety}

Dexamethasone was well tolerated with intrathecal chemotherapy and ketamine sedation. No patient was admitted to the hospital after the procedure for any reasons. No clinically significant dexamethasone side effects were observed in this study.

\section{Discussion}

Routine prophylactic administration of antiemetic agents to children with leukemia undergoing intrathecal chemotherapy and ketamine sedation is controversial at present. Previous studies of intrathecal chemotherapy have reported a wide range of vomiting incidence (10\% to $88.5 \%$ ) $[9,10]$. The present study revealed that the incidence of vomiting and nausea is $31.8 \%$ and $62.1 \%$, respectively. Jayabose et al. [9] reported only one of 52 children who had 228 lumbar punctures, experienced vomiting. However, our study was based on the use of propofal, which is low in emetogencity $[10,11]$. In contrast, the pediatric oncology sedation trial showed that during the first 12 hours following intrathecal chemotherapy and propofal sedation, there was a higher incidence of nausea and vomiting. However, patients experienced significantly fewer episodes of vomiting or retching (overall reduction of 4.66, $\mathrm{p}<0.001$ ) after receiving ondansetron [12]. In addition, Parker et al. [13] described the incidence of vomiting after receiving ketamine sedation for intrathecal chemotherapy to be $88.5 \%$ despite the use of intravenous ondansetron. However, their study did not clearly define the incidence of vomiting and nausea and separate the two entities. Interestingly, similar to our findings, they also found increased vomiting associated with decreasing patient age. The clinical significance of this finding remains to be determined.

The sedative agent, ketamine, provides amnesia and analgesia, with the side effect of vomiting ranging from $10 \%$ to $26 \%$ [14]. Langston et al. [4] investigated 268 patients who were randomized to placebo or ondansetron $0.15 \mathrm{mg} / \mathrm{kg} /$ dose as antiemetic prophylaxis before received intravenous ketamine $1 \mathrm{mg} / \mathrm{kg} / \mathrm{dose}$ for procedural sedation in the emergency department. Intravenous ondansetron significantly reduced the incidence of vomiting to $7.8 \%$ compared to $18.9 \%$ in the placebo group. However, the number needed to treat to prevent one episode of vomiting was nine. They concluded that intravenous ondansetron improved vomiting in this situation but the high cost of ondansetron limits clinical utility.

In the setting of intrathecal chemotherapy and ketamine sedation, the high cost of ondansetron is a significant factor to limit its use. Dexamethasone was first reported to be an effective prophylactic antiemetic agent in patients receiving chemotherapy $[15,16]$. The etiology and mechanism by which dexamethasone 
reduces both nausea and vomiting are not fully understood. Possibly, the effects are centrally mediated through inhibition of both prostaglandin synthesis and endogenous opioid release [17]. Glucocorticoids may be equal to or better than other drugs such as ondansetron, or metoclopramide in preventing nausea and vomiting associated with chemotherapy and post procedural sedation $[18,19]$. Ondansetron and dexamethasone have similar efficacy when administered for antiemetic prophylaxis in adults [20]. Subramanium et al. [21] reported on 135 children undergoing strabismus repair using intravenous dexamethasone $1 \mathrm{mg} / \mathrm{kg}$ or intravenous ondansetron $100 \mathrm{mg} / \mathrm{kg}$ as prophylaxis for anti-emesis. The incidence of nausea and vomiting was significantly less in the dexamethasone group. It was also shown that the cost benefit ratio was 22 times higher in ondansetron group than in the dexamethasone group.

Parker et al. [13] reported the efficacy of intravenous ondansetron in preventing vomiting after the administration of intrathecal chemotherapy in children. They included 26 children ages 18 months to 15 years receiving intrathecal chemotherapy with either metrotrexate or the combination of metrotrexate, cytarabine, and hydrocortisone. The study was a double blinded, randomized and crossover design with patients receiving an infusion of either normal saline or ondansetron 0.15 or $0.45 \mathrm{mg} / \mathrm{kg}$. The absolute risk reduction of vomiting by ondansetron was $48.1 \%$. The number needed to treat was 2.5 . This finding was similar to our study, which showed the absolute risk reduction of vomiting by dexamethasone was $33.3 \%$. The number needed to treat was three to prevent one episode of vomiting. Our results also showed a marked reduction in mild, moderate, and severe nausea between the two treatment groups. The dexamethasone group had a $45.4 \%$ incidence of some degree of nausea compared to the placebo group (78.8\% incidence). The problem of period, carryover, and sequence effects are major challenges in interpreting outcomes from any crossover study. Our data did not show any of these effects. This was probably due to the short half-life of intrathecal chemotherapy and ketamine agents along with the adequate washout period of at least two weeks in our study [22, 23]. Our results revealed the mean time to vomiting was 54 minutes in the dexamethasone group and 57 minutes in the placebo group. This was shorter than a previous study by Holdworth et al. [6] where nausea and vomiting typically began approximately three to four hours after intrathecal chemotherapy. However, that study did not indicate which sedative agents were used. Ketamineassociated vomiting almost always occurs after the recovery phase when the patient is alert [24]. We believe that the time to vomiting in our study is short because of the use of ketamine.

We used ondansetron as the standard rescue agent for vomiting in our study. Interestingly, the response rate of vomiting after receiving ondansetron in dexamethasone group was $100 \%$, whereas the response rate of vomiting after receiving ondansetron in placebo group was less (68\%). The combination of prophylactic steroid and ondansetron is superior than using single agent ondansetron for some patients. Several controlled clinical trials substantiate the combination of a corticosteroid with ondansetron being more effective than monotherapy for prophylaxis against chemotherapy induced nausea and vomiting and postoperative nausea and vomiting [25, 26].

There were no clinically significant complications after intravenous dexamethasone in this study. However, the safety conclusions were limited given the small sample size. Long-term use of prophylactic steroids may cause significant problems, such as the potential increased risk of infection and osteonecrosis. However, no studies have identified serious complications associated with antiemetic doses of dexamethasone, with a meta-analysis even indicating insufficient power to detect rare complications [27].

In our study, dexamethasone at $0.25 \mathrm{mg} / \mathrm{kg}$ administered as a single intravenous infusion before intrathecal chemotherapy could significantly reduce both the frequency of vomiting and nausea after intrathecal chemotherapy and ketamine sedation. There is no accepted standard dose for intravenous dexamethasone in this context. Madan et al. [28] reported using prophylactic intravenous dexamethasone at a dose of $0.25 \mathrm{mg} / \mathrm{kg}$. Two studies confirmed the effectiveness of the low-dose intravenous dexamethasone of $0.15 \mathrm{mg} / \mathrm{kg}$ in preventing nausea and vomiting after tonsillectomy and strabismus correction in children [29, 30]. The information about oral dexamethasone for prevention vomiting in children is limited. Ng et al. [31] reported 81 patients who received anthracycline and carboplatin. Orally co-administration of $8 \mathrm{mg}$ of ondansetron plus $8 \mathrm{mg}$ of oral dexamethasone achieved control of acute vomiting in $75 \%$ of all patients receiving chemotherapy. Their study concluded that 
the benefits of using oral anti-emetics include reduction in drug costs and nursing time. Oral dexamethasone administration may also be possibly used as a prophylactic medication with intrathecal chemotherapy and ketamine sedation, particularly when given at home. Further research in the use of oral corticosteroids may be useful.

\section{Conclusion}

Children who received intravenous dexamethasone $0.25 \mathrm{mg} / \mathrm{kg}$ have a significant reduction of vomiting and nausea after sedation with intravenous ketamine and intrathecal chemotherapy administration when compared to placebo. The combination of low cost and high efficacy makes dexamethasone a reasonable option for prophylaxis against nausea and vomiting in this patient population.

\section{Acknowledgement}

This study has approved and supported for funding from Phramongkutklao College of Medicine in 2009. The authors have no conflict of interest to declare.

\section{References}

1. Onciu M. Acute lymphoblastic leukemia. Hematol Oncol Clin North Am. 2009; 23:655-74.

2. Pui CH, Campana D, Pei D, Bowman WP, Sandlund JT, Kaste SC, et al. Treating childhood acute lymphoblastic leukemia without cranial irradiation $\mathrm{N}$ Engl J Med. 2009; 360:2730-41.

3. Holdsworth MT, Raisch DW, Winter SS, Chavez CM. Assessment of the emetogenic potential of intrathecal chemotherapy and response to prophylactic treatment with ondansetron. Support Care Cancer. 1998; 6:132-8.

4. Langston WT, Wathen JE, Roback M, Bajaj L. Effect of ondansetron on the incidence of vomiting associated with ketamine sedation in children: a double-blind, randomized, placebo-controlled trial. Ann Emerg Med. 2008; 52:30-4.

5. Jordan K, Kasper C, Schmoll HJ. Chemotherapyinduced nausea and vomiting: Current and new standards in the antiemetic prophylaxis and treatment. Eur J Cancer. 2005; 41:199-205.

6. Holdsworth MT, Raisch DW, Duncan MH, Chavez CM, Leasure MM. Assessment of chemotherapy-induced emesis and evaluation of a reduced-dose intravenous ondansetron regimen in pediatric outpatients with leukemia. Ann Pharmacother. 1995; 29:16-21.

7. Jones B, Kenward MG. Design and Analysis of CrossOver Trials. $1^{\text {st }}$ ed. Chapman \& Hall/CRC. 1989; p. 340.
8. Senn SS. Cross-over Trials in Clinical Research. $2^{\text {nd }}$ ed. Chichester:John Wiley \& Sons. 2002; p. 345.

9. Jayabose S, Levendoglu-Tugal O, Giamelli J, Grodin W, Cohn M, Sandoval C, et al. Intravenous anesthesia with propofol for painful procedures in children with cancer. J Pediatr Hematol Oncol. 2001; 23:290-3.

10. Larsson S, Argeirsson B, Magnusson J. Propofolfentanyl anesthesia compared to thiopental-halothane with special reference to recovery and vomiting after pediatric strabismus surgery. Acta Anaesthsiol Scand. 1992; 36:182-6.

11. Martin TM, Nicholson SC, Bargas MS. Propofol anesthesia reduces emesis and airway obstruction in pediatric outpatients. Anesth Analg. 1993; 76:144-8.

12. Nagel K, Willan AR, Lappan J, Korz L, Buckley N, Barr RD. Pediatric oncology sedation trial (POST): A double-blind randomized study. Pediatr Blood Cancer. 2008; 51:634-8.

13. Parker RI, Prakash D, Mahan RA, Giugliano DM, Atlas MP. Randomized, double-blind, crossover, placebo-controlled trial of intravenous ondansetron for the prevention of intrathecal chemotherapy-induced vomiting in children. J Pediatr Hematol Oncol. 2001; 23: 578-81.

14. Pitetti RD, Singh S, Peirce MC. Safe and efficacious use of procedural sedation and analgesia by non anesthesiologists in a pediatric emergency department. Arch Pediatr Adolesc Med. 2003; 157:1090-6.

15. Aapro MS, Alberts DS. Dexamethasone as an antiemetic in patients treated with cisplatin. N Engl J Med. 1981; 305:520.

16. Markman M, Sheidler V, Ettinger DS, Quaskey SA, Mellits ED. Antiemetic efficacy of dexamethasone. Randomized, double-blind, crossover study with prochlorperazine in patients receiving cancer chemotherapy. N Engl J Med. 1984; 311:549-52.

17. Holte K, Kehlet H. Perioperative single-dose glucocorticoid administration: pathophysiologic effects and clinical implications. J Am Coll Surg. 2000; 195: 694-712.

18. Olver I, Clark-Snow RA, Ballatori E, Espersen BT, Bria E, Jordan K. Guidelines for the control of nausea and vomiting with chemotherapy of low or minimal emetic potential. Support Care Cancer. 2011; 19:33-6.

19. Bisgaard T, Klarskov B, Kehlet H, Rosenberg J. Preoperative dexamethasone improves surgical outcome after laparoscopic cholecystectomy: a randomized double-blind placebo-controlled trial. Ann Surg. 2003; 238:651-60.

20. Apfel CC, Korttila K, Abdalla M, Kerger H, Turan A, 
Vedder I, et al. A factorial trial of six interventions for the prevention of postoperative nausea and vomiting. N Engl J Med. 2004; 350:2441-51.

21. Subramaniam B, Madan R, Sadhasivam S, Sennaraj B, Tamilselvan P, Rajeshwari S, et al. Dexamethasone is a cost-efective alternative to ondansetron in preventing PONV after paediatric strabismus repair. Br J Anaesth. 2001; 86:84-9.

22. Hesketh PJ, Kris MG, Grunberg SM, Beck T, Hainsworth JD, Harker G, et al. Proposal for classifying the acute emetogenicity of cancer chemotherapy. J Clin Oncol. 1997; 15:103-9.

23. Tanihata S, Oda S, Nakai S, Uchiyama T. Antiemetic effect of dexamethasone on cisplatin-induced early and delayed emesis in the pigeon. Eur J Pharmacol. 2004; 484:311-21.

24. Green SM, Krauss B. Clinical practice guideline for emergency department ketamine dissociative sedation in children. Ann Emerg Med. 2004; 44:460-71.

25. Janinis J, Giannakakis T, Athanasiades A, Fountzilas G, Bafaloukos D, Kosmidis P, et al. A randomized open-label parallelgroup study comparing ondansetron with ondansetron plus dexamethasone in patients with metastatic breast cancer receiving high-dose epirubicin. A Hellenic Cooperative Oncology Group study. Tumori. 2000; 86:37-41.

26. Lopez-Olaondo L, Carrascosa F, Pueyo FJ, Monedero P, Busto N, Saez A. Combination of ondansetron and dexamethasone in the prophylaxis of postoperative nausea and vomiting. Br J Anaesth. 1996; 76: 835-40.

27. Henzi I, Walder B, Tramer MR. Dexamethasone for the prevention of postoperative nausea and vomiting: a quantitative systematic review. Anesth Analg. 2000; 90:186-94.

28. Madan R, Bhatia A, Chakithandy S, Subramaniam R, Rammohan G, Deshpande $\underline{S}$, et al. Prophylactic dexamethasone for postoperative nausea and vomiting in pediatric strabismus surgery: a dose ranging and safety evaluation study. Anesth Analg. 2005; 100:1622-6.

29. Liechti M, Feurer R, Gross D, Schmitz A, Stutz K, Gerber A, et al. Prevention of postoperative nausea and vomiting in children following adenotonsillectomy, using tropisetron with or without low-dose dexamethasone. J Anesth. 2007; 21:311-6.

30. Mathew PJ, Madan R, Subramaniam R, Bhatia A, Mala CG, Soodan A, et al. Efficacy of low-dose dexamethasone for preventing postoperative nausea and vomiting following strabismus repair in children. Anaesth Intensive Care. 2004; 32:372-6.

31. Ng WL, Della-Fiorentina SA. The efficacy of oral ondansetron and dexamethasone for the prevention of acute chemotherapy-induced nausea and vomiting associated with moderately emetogenic chemotherapya retrospective audit. Eur J Cancer Care (Engl). 2010; 19:403-7. 